\title{
Level of chemical and microbiological contaminations in chili bo (Paste).
}

\begin{abstract}
The objective of this study was to determine the level of preservatives and microbiological loads in various brands of commercially available chili bo (paste). Fifteen different brands of chili bo obtained from the local market and hypermarkets were analyzed for $\mathrm{pH}$, moisture and benzoic acid content, microbiological loads (aerobic, anaerobic, aerobic spores, and fungi), and thermophilic microorganisms. Results showed that both moisture content and $\mathrm{pH}$ vary among samples. The concentrations of benzoic acid detected in chili bo were found to be in the range of 537 to $5,435 \mathrm{mg} / \mathrm{kg}$. Nine of fifteen brands were found to exceed the maximum level permitted by the Malaysian Food Law in accordance with the Codex Alimentarius $(1,000 \mathrm{mg} / \mathrm{kg}$ for benzoic acid). An apparent correlation between benzoic acid concentration and microbiological loads present in the chili bo was observed. The microbiological loads were found to be relatively low in the end products containing high amounts of benzoic acid. The heat-resistant (70 to 80 degrees C) microorganisms present in chili bo were identified as Ochrobacterum tritici, Stenotrophomonas rhizophila, Microbacterium maritypicum, Roseomonas spp., CDC group II-E subgroup A, Flavimonas oryzihabitans, and Pseudomonas aeruginosa, with M. maritypicum being the most frequently found (in 9 of 15 samples) microorganism. Most of these identified microorganisms were not known to cause foodborne illnesses.
\end{abstract}

Keyword: Chilli Boh; Chemical contaminants; Microbiological contaminants; Food safety. 